$10.2478 / \mathrm{gb}-2020-0010$

sciendo

\title{
Brücke, Grenzstein, Smaragd... Metaphorische Darstellungen Schlesiens und seiner Bewohner
}

\section{Monika CZOK}

Dr.; Universität Opole, Polen; E-Mail: czok.monika@gmail.com

\begin{abstract}
The article shows a metaphoric portrayal of the Silesia region. The author analyses metaphors which are being used to describe and characterize this region, namely Silesia as a bridge, a boundary stone, a pear tree, a smaragd and other figures. The analysis shows that the metaphors express the multicultural character of Silesia. They are predominantly very positive assessed.
\end{abstract}

Furthermore, the author analyses metaphors which are being used to characterize the inhabitants of Silesia. The analysis shows that this metaphors express a positive as well as a critical perception of this population.

Keywords: Silesia, Upper Silesia, metaphor, bridge

Schlesien ist eine Region im Südwesten Polens ${ }^{1}$. Im Laufe der Geschichte, beinahe alle zwei Jahrhunderte, wechselte die $\mathrm{Zu}-$ gehörigkeit zu jeweils anderen Herrschaftsgebieten. Zunächst gehörte Schlesien zum Großmährischen Reich, wurde dann vom böhmischen Herzog, daraufhin von den polnischen und danach schlesischen Piasten regiert. Schlesien wurde Teil des Heiligen Römischen Reiches. 1335-1526 war es unter der böhmischen Krone, 1526-1742 unter dem Hause Habsburg. Nach den so genannten Schlesischen Kriegen gehörte der größte Teil Schlesiens

${ }^{1}$ Ein kleiner Teil im Westen der früheren preußischen Provinz Niederschlesien gehört zu Deutschland, ein südlicher Teil von Oberschlesien zu Tschechien. 
zu Preußen und damit von 1871 bis 1945 zum Deutschen Reich. Eine virulente Geschichte erlebte Schlesien in der Zwischenkriegszeit, als die eine Region zwischen drei Staaten aufgeteilt wurde. Nach dem Zweiten Weltkrieg wurde Schlesien größtenteils unter polnische Verwaltung gestellt (de facto: in den polnischen Staat eingegliedert). Wegen seiner topographischen Lage war und ist Schlesien ein Grenzgebiet. Gegenwärtig ist es ein Grenzgebiet im direkten (administrativen) Sinne, doch noch vielmehr ist es ein mentales Grenzgebiet. Hier lebt der zahlenmäßig größte Teil der deutschen Minderheit in Polen. Neben Polnisch als Amts- und Hauptsprache wird Deutsch als Minderheitensprache (teilweise mit zweisprachigen Ortsschildern und der Möglichkeit des Unterrichts von Deutsch als Muttersprache in den Schulen) und Schlesisch als Dialekt gesprochen. In seiner Geschichte war Schlesien nicht nur mehrsprachig, sondern auch mehr-konfessionell (heute spielen andere Konfessionen als die katholische eher eine marginale Rolle) und multikulturell. All diese Faktoren tragen dazu bei, dass Schlesien als eine spezifische, eine einzigartige Region bezeichnet wird.

Die Autoren, die sich literarisch oder wissenschaftlich mit Schlesien befassen, werden somit mit der Herausforderung konfrontiert, der Spezifik und Komplexität dieser Region Ausdruck zu geben. Die Analyse der Literatur aus oder über Schlesien zeigt, dass dabei oftmals zu metaphorischen Figuren gegriffen wird.

Innerhalb der Schlesien beschreibenden Metaphern stellt die Versinnbildlichung Schlesien als Brücke die populärste Metapher schlechthin dar. Nach dem Ersten Weltkrieg entstanden, ${ }^{2}$ erlebt diese Metapher nach der Wende von 1989 und den

2 Vgl. Conrads, der die Geschichte der Metapher Schlesien als Brückenlandschaft in seinem voluminösen Werk Schlesien beschreibt. Er platziert den Ursprung dieses Motivs in die Zeit nach dem Ende des Ersten Weltkrieges, als Schlesien infolge der Bestimmungen des Versailler Vertrages vor eine Gebietsaufteilung gestellt wurde. Die Teilung der bis dahin einheitlichen Region und die Abgabe ihrer Gebiete an den 
veränderten politischen und gesellschaftlichen Umständen eine Wiedergeburt. Auf diese Figur wird (besonders in den ersten Jahren nach der Wende) gerne in den Buchtiteln und Konferenzen zurückgegriffen. ${ }^{3}$ In erster Linie wird (Ober-)Schlesien die Brückenfunktion zwischen den beiden Staaten Polen und Deutschland sowie deren Bevölkerung zugeschrieben. Dem Bild der Brücke werden ferner weitere Bedeutungen beigemessen, wie z.B. die Brücke zwischen Polen, Deutschland und Tschechien zugleich, die Brücke zu Europa, die Brücke zwi-

neuen polnischen Staat sowie an die neu gebildete Tschechoslowakei verursachten, dass die Verbindung zu den übrigen Teilen Deutschlands bedroht schien. Der am östlichen Rande des deutschen Staates gelegenen Region wurde die Rolle der Brücke zugewiesen. Conrads betont die pejorative Aussage dieses Symbols aus seiner Entstehungszeit: „Wenn man (...) von der Brücke Schlesien sprach, so verstanden (...) viele darunter eine Art Verteidigungswerk. Dieses abweisende Brückenbild war die Folge ethnischer Auseinandersetzungen mit den slawischen Nachbarn und wollte zunächst der isolierten Lage Schlesiens Ausdruck verleihen. Die Brücke verband keine Nachbarn und Völker mehr, sondern hatte nur noch eine auf das Deutschtum bezogene Klammerfunktion." (Conrads, Norbert: Silesiographia oder Landesbeschreibung. In: Ders.: Deutsche Geschichte im Osten Europas. Schlesien. Berlin, 1994, S. 13-36.)

${ }^{3}$ Einige Beispiele: Koschyk, Hartmut: Euroregion Schlesien - eine Brücke zwischen Deutschen und Polen. In: Heinrich Trierenberg (Hrsg.): Schlesien heute. Eine Brücke zwischen Deutschen und Polen. Leer 1991, S. 10-21; Oberschlesien als Brücke zwischen Polen und Deutschen. Symposium vom 8.-9. Mai 1990 in Opole. Mülheim/Ruhr 1990, polnische Fassung: Berlińska, Danuta/ Lesiuk, Wiesław/ Śmiełowska, Maria: Górny Śląsk jako pomost pomiędzy Polakami $i$ Niemcami: V Sympozjum: materiaty strony polskiej na V Sympozjum. Opole 1990; Brehmer, Dietmar: Mniejszość niemiecka na Górnym Śląsku. Pomost między Polakami a Niemcami? In: Mniejszości na Górnym Ślasku. Pomost czy przeszkoda w stosunkach polsko-niemieckich? Materiaty polsko-niemieckiego sympozjum odbytego $w$ Mülheim/R. w dniach 2-4 grudnia 1993 r. Unter der Leitung von Dieter Bach und Krystian Heffner, wiss. Red. von Wiesław Lesiuk, Opole1994, S. 62-67. 
schen Westen und Osten. Einige dieser Aspekte werden im Folgenden thematisiert. ${ }^{4}$

\section{Brücke zwischen Polen und Deutschland}

Die geographische Lage Schlesiens sowie seine historische wie gegenwärtige Bindung an die beiden Staaten Deutschland und Polen führt dazu, dass das Motiv der Brücke in Bezug auf diese Region immer wieder aufgegriffen wird.

In der gegenwärtigen Darstellung der Geschichte Schlesiens wird der Aspekt der Brückenfunktion in Bezug auf die beiden Länder deutlich hervorgehoben. Gern wird dabei auf die Historizität dieser Brückenrolle hingewiesen, wie etwa bei Bieniasz: „Jahrhundertelang war Schlesien eine Brücke, auf der Polen und Deutsche sich begegneten. ${ }^{\text {"5 }}$ Ebenfalls will die Leitung des Landesmuseums Schlesien in Görlitz auf diese Historizität und die „Natürlichkeit“ dieser Rolle hindeuten:

Die geographische Lage und Gestalt am Ober- und Mittellauf der Oder hat Schlesien schon immer eine Schlüsselstellung und eine natürliche Mittlerfunktion zwischen West und Ost, Nord und Süd zugewiesen.

Schlesien hat daher als Brücken- und Durchgangslandschaft im Laufe seiner Geschichte stets Einfluss von allen Seiten erfahren, so

${ }^{4} \mathrm{Zu}$ einer ausführlicheren Analyse dieser und weiterer Aspekte wird auf folgenden Beitrag der Autorin hingewiesen: Czok, Monika: Schlesien als Brücke - aber wohin? Vielfache Dimensionen einer äußerst beliebten Metapher. In: Jokiel, Małgorzata/ Pelka, Daniela (Hrsg.): Germanistische Werkstatt 9. Opole 2020 (in Druck). Ferner sei auf die Dissertation der Autorin hingewiesen, in der die Schlesien beschreibenden Metaphern in Bezug auf Fremd- und Selbstzuschreibungen kontextualisiert werden. (Czok, Monika: Das Oppelner Schlesien und seine Identität. Diskurse - Sozial-politisches - regionale Presse. Wrocław 2017. [Manuskript]

5 Bieniasz, Stanisław: Schlesien - die dritte Option. In: Deutsche und Polen. 100 Schlüsselbegriffe. Hrsg. v. Ewa Kobylińska, Andreas Lawaty, Stephan Rüdiger. München 1992, S. 540-547, hier S. 541. 
wie es sich selbst in umgekehrter Richtung in das kulturelle Geschehen der Nachbarländer einbrachte. ${ }^{6}$

Als „natürlich“ versteht es ferner Wiesław Lesiuk, der die Brückenfunktion Schlesiens folgendermaßen begründet: ,Diese Rolle resultiert sowohl aus den historischen Gegebenheiten als auch aus der gegenwärtigen ethnischen Situation dieser Region. Das Oppelner Schlesien lag immer auf der Schnittstelle dreier Kulturen: der polnischen, deutschen und böhmischen." ${ }^{\text {"7 }}$ Dabei dehnt er das Bild Schlesiens als Brücke auf ein drittes Land aus, nämlich als Brücke zwischen Polen, Deutschland und Tschechien zugleich.

\section{Brücke zur Verständigung zwischen den Völkern}

Das oben erwähnte Zitat von Bieniasz führt in eine weitere Dimension des deutsch-polnischen Brückenbildes: Von der Ebene zweier Staatsgebilde auf die Ebene der zwischenmenschlichen Verhältnisse. Nach der Wende wurde die Brückenfunktion Schlesiens vor allem im Hinblick auf die deutsch-polnischen Beziehungen genutzt.

In einigen Texten wird dies als ein Wunsch ausgedrückt: „In der Predigt aus Anlaß des gemeinsamen Gottesdienstes knüpfte Bischof Nossol von Oppeln an die guten Traditionen dieses symbolträchtigen Ortes Kreisau an, in der Hoffnung, daß ganz Schlesien, eine Brückenfunktion bei der Festigung und

${ }^{6}$ Schlesien - eine Brücke in Europa. Aspekte einer Kulturlandschaft in der Mitte Europas. Hg. Vom Landesmuseum Schlesien in Görlitz. Görlitz 1993, S. 5.

${ }^{7}$ Lesiuk, Wiesław: Czy Śląsk Opolski może być przykładem regionu na drodze do Europy? In: Ders. (Hrsg.): Region jako przyszła struktura europejska. Materiaty polsko-niemieckiego sympozjum odbytego w Opolu $w$ dniach 13-15 maja 1992 r. Unter der Leitung von Dieter Bach und Krystian Heffner. Opole 1992, S. 112-115. 
Aussöhnung zwischen unseren beiden Völkern zukommen' werde. "“8; in anderen Texten dagegen wird die Rolle der Brücke als ein Stand der Dinge präsentiert. Demzufolge bilde Oberschlesien eine Brücke für ,das Zusammenkommen von Polen und Deutschen" , für die Verständigung sowie für die Versöhnung zwischen beiden Nationen. Der Autor dieser Worte vermerkt, dass innerhalb Oberschlesiens besonders das Oppelner Schlesien ${ }^{10}$ als Brücke zu betrachten sei. Er sieht die Begründung für die Brückenrolle in dem historischen Kontext der Emigration und den daraus resultierenden Verbindungen unter der

8 Conrads, Norbert: Silesiographia oder Landesbeschreibung. In: Ders.: Deutsche Geschichte im Osten Europas. Schlesien. Berlin1994, S. 1336, hier S. 29.

9 Kroszel, Janusz: Einführung. In: Oberschlesien als Brücke zwischen Polen und Deutschen. Symposium vom 8.-9. Mai 1990 in Opole. Mülheim/Ruhr 1990. S. 3-9, hier S. 3.

${ }^{10}$ Das Oppelner Schlesien ist ein Teil des historischen Oberschlesiens. Der Name geht auf die Teilung Oberschlesiens im Jahre 1921 zurück, als Oberschlesien in das zum polnischen Staat gehörende Gebiet (sog. Ostoberschlesien) und in das bei dem deutschen Staat verbliebene Gebiet geteilt wurde, das in der polnischen Sprache Ślask Opolski, das Oppelner Schlesien, genannt wurde. Der Name hat sich in dem polnischen Begriffsapparat etabliert und wird heute allgemein verwendet. Infolge der historischen Ereignisse der letzten Jahrzehnte ist zwischen dem Oppelner Schlesien und dem östlichen Teil Oberschlesiens (auch als das sog. Kattowitzer Schlesien bezeichnet) eine faktische (administrative) und mentale Spaltung zu verzeichnen, sodass in dem aktuellen polnischen Diskurs des Öfteren zwischen den beiden Subregionen unterschieden wird. Das Problem der Auffassung dieses Raumes (Śląsk Opolski als Subregion Oberschlesiens oder als eigenständige, von Oberschlesien getrennte Region) ist zum Gegenstand der Doktorarbeit der Autorin geworden. Siehe: Czok, Monika: Das Oppelner Schlesien und seine Identität. Diskurse - Sozial-politisches - regionale Presse. Wrocław 2017 [Manuskript]. Ferner ist auf folgenden Beitrag hinzuweisen: Joachimsthaler, Jürgen: Die mehrfach erfundene Provinz. Schlesien zwischen Entwurf und Wirklichkeit. In: Schmitz, Walter/ Joachimsthaler, Jürgen (Hrsg.): Verhandlungen der Identität. Literatur und Kultur in Schlesien seit 1945. Dresden 2004, S. 109-135. 
Bevölkerung beider Länder. Der Autor will es ,als Gebiet [...] sehen, in dem sich beide Völker näher kommen können, in dem die Chance des Kennenlernens und der Verständigung in besonderer Weise genutzt werden kann. “11

Auch Teresa Sołdra-Gwiżdż thematisiert die Rolle des Oppelner Schlesiens als Brücke - in ihrer Auffassung als „Brücke zwischen zwei benachbarten Völkern“12, also mit der Betonung des nachbarschaftlichen Verhältnisses.

Die Metapher der Brücke wird in Oberschlesien ferner auf die Gruppe der deutschen Minderheit bezogen, die dort am zahlenstärksten in Polen vertreten ist. Die Brückenfunktion der deutschen Minderheit geht auf den Nachbarschaftsvertrag von 1991 zurück, ${ }^{13}$ in dem der rechtliche Status der deutschen Minderheit geklärt wurde und ihre Rechte niedergeschrieben wurden. Im Vertrag wurde der deutschen Minderheit die Rolle der „natürlichen Brücke zwischen dem deutschen und dem polnischen Volk“ zugeschrieben. Innerhalb der drei Jahrzehnte der Aktivität der organisierten ${ }^{14}$ deutschen Minderheit in der Region

${ }^{11}$ Kroszel, Janusz: Einführung. In: Oberschlesien als Brücke zwischen Polen und Deutschen. Symposium vom 8.-9. Mai 1990 in Opole. Mülheim/Ruhr 1990, S. 3-9, hier S. 3.

${ }^{12}$ Sołdra-Gwiżdż, Teresa: Wielokulturowość Śląska Opolskiego i jego młodego pokolenia w perspektywie socjologicznej. In: Wiesław Lesiuk (Hrsg.): Region jako przyszła struktura europejska. Materiały polsko-niemieckiego sympozjum odbytego w Opolu $w$ dniach 13-15 maja 1992 r., pod kierownictwem Dietera Bacha i Krystiana Heffnera. Opole1992, S. 45-52, hier S. 45.

${ }^{13}$ Vertrag zwischen der Bundesrepublik Deutschland und der Republik Polen über gute Nachbarschaft und freundschaftliche Zusammenarbeit vom 17. Juni 1991, unter: https://polen.diplo.de/blob/484312/b6dba5 $931 \mathrm{~d} 46 \mathrm{bfdbaf72}$ f8dff299a839/vertrag-17-6-1991-data.pdf [13.03.2020].

${ }^{14}$ In erster Linie sind das die Sozial-Kulturelle Gesellschaft der Deutschen im Oppelner Schlesien, DFK Schlesien sowie der Verband der deutschen Sozial-Kulturellen Gesellschaften in Polen mit Sitz in Oppeln. Auf ihre Struktur, Geschichte und Zielsetzung sei auf die einschlägige, sehr umfangreiche Literatur hingewiesen. 
wurde mit der Brückenmetapher unterschiedlich umgegangen, sowohl durch die Mitglieder der Gruppe selbst als auch durch die äußeren Akteure; die Aussagen reichen von hurraoptimistischen bis gar zu spöttischen Tönen. ${ }^{15}$

\section{Brücke zu Europa}

In räumlicher Dimension wird die Versinnbildlichung Schlesien als Brücke weiter gestreckt. In der Auffassung des ehemaligen Bischofs der Diözese Oppeln und Initiators des deutsch-polnischen Versöhnungsprozesses ${ }^{16}$ Alfons Nossol erstreckt sich die Rolle Schlesiens als Brücke über die einzelnen Staaten hinweg, sie erhält eine europäische Dimension: „Das kulturelle Merkmal Schlesiens als Brücke besitzt heute eine besondere Bedeutung für unseren ganzen Kontinent [...]. Das sich vereinende Europa

${ }^{15}$ Zur detaillierten Analyse dieses Aspektes sei auf folgenden Beitrag hingewiesen: Czok, Monika: Schlesien als Brücke - aber wohin? Vielfache Dimensionen einer äußerst beliebten Metapher. In: Małgorzata Jokiel, Daniela Pelka (Hrsg.): Germanistische Werkstatt 9. Opole 2020 (im Druck). Über die Selbstauffassung der deutschen Minderheit im Oppelner Schlesien sowie ihre Wahrnehmung von außen sei auf die Dissertation der Autorin hingewiesen. Vgl. dazu Czok, Monika: Das Oppelner Schlesien und seine Identität. Diskurse - Sozial-politisches - regionale Presse. Wrocław 2017, S.171-212 [Manuskript].

${ }^{16}$ Auf ihn geht u.a. die Initiative der deutsch-polnischen Versöhnungsmesse in Kreisau zurück. Außerdem hat er ,sich sein Leben lang mit Nachdruck und persönlichem Einsatz für einer Brückenschlag zwischen den Menschen unterschiedlicher Herkunft, Nationalität und verschiedenen Glaubens eingesetzt.“ Er setzte sich für die „Verständigung und Aussöhnung zwischen der polnischen Mehrheit und deutschen Minderheit" in Schlesien ein. Aus diesen und weiteren Gründen wird er selbst als ,einer der großen Brückenbauer in Europa“ bezeichnet. (Alle Zitate aus der Begründung für die Verleihung des Internationalen Brückenpreises der Europastadt Görlitz/Zgorzelec an Erzbischof em. Prof. Dr. Alfons Nossol, unter: http://www.brueckepreis.de/?ID=113 \&art_param=17 [1.09.2019].) 
als eine Gemeinschaft des Geistes, das heißt eine Gemeinschaft der Kultur und der Werte, braucht heute Brücken"17.

Die Metapher der Brücke ist bei Nossol eine oftmals verwendete Figur. Hierbei ist er jedoch keine Ausnahme; zahlreiche weitere Autoren betrachten Schlesien als eine Brücke zu Europa. Diese Auffassung war vor allem in den 90er und frühen 2000er Jahren, also noch vor dem EU-Beitritt Polens, populär.

So beendet Conrads seine Ausführungen über die Geschichte der Brückenmetapher in Bezug auf Schlesien mit der zukunftsgerichteten Prognose (das Werk wurde 1994 herausgegeben): „Besonders Oberschlesien habe heute die Chance, eine Brückenfunktion (...) zwischen Polen und Europa zu übernehmen."18 Im ähnlichen Sinne schließt Bieniasz seinen Essay über Schlesien mit einer zukunftsorientierten Hoffnung, dass „Oberschlesien eine Brückenfunktion zwischen Polen und Deutschland sowie zwischen Polen und Europa übernimmt" ${ }^{\text {" }}{ }^{19}$ Die Brückenrolle „auf dem Weg nach Europa“ klingt auch in der schon oben erwähnten Auffassung von Lesiukan. ${ }^{20}$ Selbst die erste Ausstellung des sich damals noch im Aufbau befindenden Schlesischen

${ }^{17}$ Nossol, Erzbischof Alfons: Schlesien, Śląsk, Slezsko! In: Hałub, Marek; Weber, Matthias (Hrsg.): Mein Schlesien - meine Schlesier. Zugänge und Sichtweisen (= Schlesische Grenzgänger, Bd. 6). Leipzig 2014, S. 123-129, hier S. 127.

${ }^{18}$ Conrads, Norbert: Silesiographia oder Landesbeschreibung. In: Ders.: Deutsche Geschichte im Osten Europas. Schlesien. Berlin 1994. S. 13-36, hier S. 29.

${ }^{19}$ Bieniasz, Stanisław: Schlesien - die dritte Option. In: Deutsche und Polen. 100 Schlüsselbegriffe. Hrsg. von Ewa Kobylińska, Andreas Lawaty, Rüdiger Stephan. München 1992, S. 540-547, hier S. 546 f.

${ }^{20}$ Lesiuk, Wiesław: Czy Śląsk Opolski może być przykładem regionu na drodze do Europy? In: Ders. (Hrsg.): Region jako przyszła struktura europejska. Materiaty polsko-niemieckiego sympozjum odbytego $w$ Opolu $w$ dniach 13-15 maja 1992 r. Unter der Leitung von Dieter Bach und Krystian Heffner. Opole1992, S. 112-115. 
Museums in Görlitz bediente sich dieses Bildes und trug den Titel: „Schlesien - eine Brücke in Europa“21.

Einerseits ließe sich die obige Betrachtung Schlesiens als Brücke zu Europa nachvollziehen: Es ist eine Region, die wegen der historischen Ereignisse sehr viel mehr Kontakt mit Deutschland und Westeuropa als andere Regionen Polens hatte. Schlesien kann somit als ein Mittler gesehen werden; diese Sichtweise ist vor allem für die ersten Jahre nach der Wende nachvollziehbar, als eine Mitgliedschaft in der Europäischen Union noch sehr weit entfernt schien.

Andererseits entpuppt sich das Gedankenkonstrukt Schlesien als Brücke zu Europa oder gar als Brücke zwischen Polen und Europa als eine Kuriosität: Es ruft in Gedanken das Bild hervor, Schlesien bzw. Polen liege außerhalb Europas und müsse erst „europäisiert“ werden! Man kann diesem Bild mit einem Gegenargument widersprechen: Schlesien muss nicht zu Europa, da es doch „im Herzen Europas“ liegt. Paradoxerweise führt der Widerspruch gegen das eine Konstrukt zu einer anderen, ebenso gerne verwendeten Metapher. Etliche Autoren preisen Schlesien an, eine Brücke zu Europa zu sein, andere - im Herzen Europas zu liegen.22

Ein zweiter kritischer Gedanke, der bei der Brücke-Metapher $\mathrm{zu}$ bedenken ist, bezieht sich auf die Popularität dieser Versinnbildlichung weit über die beschriebene Region hinaus. Bei der Betrachtung unterschiedlicher Publikationen kommt

${ }^{21}$ Schlesien - eine Brücke in Europa. Aspekte einer Kulturlandschaft in der Mitte Europas. Hrsg. vom Landesmuseum Schlesien in Görlitz. Görlitz 1993, S. 5.

${ }^{22}$ Exemplarisch dazu bei: Schulz, Thomas: Zwischen Wissenschaft und Öffentlichkeit. Schlesien im Programm des deutschen Kulturforums Östliches Europa. In: Schlesische Gelehrtenrepublik. Bd. 6, 2014, S. 419-438, hier S. 425; Starbatty, Joachim/ Stühmer, Axel: Einführung in das Symposium. In: Gerken, Lüder/ Starbatty, Joachim (Hrsg.): Schlesien auf dem Weg in die Europäische Union. Ordnungspolitik der sozialen Marktwirtschaft und christliche Gesellschaftslehre. Stuttgart 2011, S. XIII. 
zum Vorschein, dass die Brückenfunktion kein Alleinstellungsmerkmal Schlesiens ist. Auch andere Regionen und Bevölkerungsgruppen schmücken sich mit dieser Eigenschaft. ${ }^{23}$ Catherine Robert bringt dieses Phänomen deutlich auf den Punkt: „Ganz Europa ist eine Brückenlandschaft. “24

\section{Weitere metaphorische Darstellungen Schlesiens}

Die Brücke ist die populärste, doch durchaus nicht einzige Metapher, auf die in Bezug auf Schlesien zurückgegriffen wird.

${ }^{23}$ So sieht sich exemplarisch die sorbische Minderheit als Brücke: „Die Sorben sehen sich im vereinten Europa als Brücke zwischen den westeuropäischen und osteuropäischen Ländern." (Schurr, Edith: Die sorbische Minderheit in der Lausitz und ihre Perspektive im vereinten Europa am Beispiel der Förderung der Zweisprachigkeit im vorschulischen Bereich, in: Stanisław Senft/ Aleksandra Trzecielińska-Polus (Hgg.): Colloquium Opole 2006: mniejszościnarodowe $i$ etniczne $w$ Polsce, Niemczech i Republice Czeskiej-tożsamość i perspektywy w jednoczacejsięEuropie, Opole 2007, S. 51-58, hier S. 58.)

${ }^{24}$ Robert, Catherine: Die deutsch-polnischen Beziehungen unter dem Aspekt der „Brückenfunktion“ und der „Kulturlandschaften “, in: Marek Hałub, Frank Stucke (Hgg): Wrocław-Berlin. Germanistischer Brückenschlag im deutsch-polnischen Dialog. II Kongress der Breslauer Germanistik. Bd. 4: Kulturwissenschaft, Wrocław, Dresden 2006, S. 289-297, hier S. 289.

Die Metapher der Brücke ist infolge des inflationären Gebrauchs zu einer Floskel geworden, was zur Folge haben kann, dass sie ihren ursprünglichen Sinn verliert.

Möglicherweise wird sie aus diesem Grund auch negativ gebraucht, wie bei Glensk: Er kommentiert die Aktivität der deutschen Minderheit nach der Wende mit Ironie: ,Was für eine Überraschung, in der direkten Nachbarschaft die e i g e n e n Deutschen zu entdecken; die selbst ein Pfeiler der Brücke seien, die nach Europa und weiter führt." (Glensk, Joachim: Organizowanie się mniejszości niemieckiej w latach 19891993 w świetle prasy regionalnej na Górnym Ślasku, in: Michał Lis (Hg.): Śląsk Opolski: nadzieje i zagrożenia demokratycznych przemian, Opole 1994, S. 76-88, hier S. 77. [Übers. MC]). 
Es sind zahlreiche Metaphern zu verzeichnen, die bei der Charakteristik der Region formuliert werden, um ihrer Multikulturalität Ausdruck zu verleihen.

Ein „Klassiker” unter den Schlesien betreffenden Metaphern, auf die sich die Autoren bei der Charakteristik Schlesiens kontinuierlich berufen, ${ }^{25}$ ist die Verbildlichung von Emil Szramek ${ }^{26}$. $\mathrm{Ihm}$ zufolge hat Oberschlesien einen Eck-Charakter, es liege am Rande des Staates und trage alle Merkmale eines Grenzgebietes. ${ }^{27}$

Szramek stellt Schlesien mittels weiterer Metaphern dar, die die Bevölkerung beschreiben und dabei auch das Land charakterisieren. Demzufolge sind die Menschen in Oberschlesien

${ }^{25}$ Z.B.: Szlachta-Misztal, Justyna: Czy istnieje kultura górnośląska? Rozważania na temat kultury regionu. In: Radomski, Andrzej/ Bomba, Radosław (Hrsg.): Granice w kulturze. Lublin 2010, S. 307-318, hier S. 308; Smolińska, Teresa: Miejsce folkloru w procesie zmian tożsamości narodowej (na przykładzie Śląska Opolskiego). In: Gaida,Waldemar u.a.: Śląsk w edukacji regionalnej. Strzelce Opolskie 2005, S. 107-121, hier S. 110; Szczepański, Marek S. (2004): Regionalizm górnośląski: los czy wybór? In: Nijakowski, Lech M. (Hrsg.): Nadciagaja Ślązacy. Czy istnieje narodowość śląska? Warszawa. S. 90-115, hier S. 104 und weitere Autoren.

${ }^{26}$ Der Gedanke vom Eckcharakter Oberschlesiens wird Szramek zugeschrieben und so findet man diese Information in den meisten Quellen. In Wirklichkeit stammt jedoch die Idee nicht von ihm, worauf Szewczyk aufmerksam macht: „Die Bezeichnung vom Eck-Charakter Oberschlesiens übernahm Szramek von einem deutschen Historiker jüdischer Abstammung, Ezechiel Zivier, dem Herausgeber der Monatsschrift Oberschlesien und Verfasser zahlreicher Bücher über die oberschlesische Industrie." (Szewczyk, Grażyna Barbara: Die Identität des Oberschlesiers im Umfeld von Ideologie und Mythos. In: Lasatowicz, Maria Katarzyna/ Joachimsthaler, Jürgen (Hrsg.): Nationale Identität aus germanistischer Perspektive. Opole1998, S. 87-99, hier S. 88).

${ }^{27}$ Szramek, Emil: Śląsk jako problem socjologiczny, zit. nach: Kijonka, Tadeusz: Po siedmiu latach kampanii.In: Lech M. Nijakowski (Hrsg.): Nadciagaja Ślazacy. Czy istnieje narodowość śląska? Warszawa 2004. S. 35-67, hier S. 63. 
„den Grenzsteinen ähnlich, die auf der einen Seite das polnische, auf der anderen Seite das deutsche Kennzeichen tragen, oder auch einem Birnbaum, der an der Grenze steht und Früchte auf beiden Seiten trägt." ${ }^{28}$ Dieses Bild Szrameks wird ebenfalls als Maßstab in Bezug auf die Charakteristik Schlesiens genommen. ${ }^{29}$

Eine Reihe von Metaphern vergleicht Schlesien mit einem Edelstein. Nossol erinnert an Heinrich Mühlpforts Verbildlichung, die Schlesien als einen Smaragd Europas darstellt: „Ein Smaragd ist ein Edelstein, der sehr kostbar ist, weil er aus unzähligen kleinen zusammengepressten Teilchen besteht, die ein Ganzes bilden." ${ }^{30}$ Die Darstellung als Edelstein hat eine durchaus vorteilhafte Ausstrahlung: Die Multikulturalität und die zahlreichen Einflüsse werden sehr positiv konnotiert. Es wird nicht auf die Herausforderungen oder gar Probleme gedeutet, die das Vorkommen unterschiedlicher Kulturen auf einem Territorium mit sich bringen kann. Im Gegenteil, die Multikulturalität und zahlreichen Einflüsse werden als der Reichtum

${ }^{28}$ Vgl. Simonides, Dorota: Mit Ślązaka, zit. nach: Ploch, Gregor/ Myszor, Jerzy/ Kucinski, Christine (Hrsg.): Die ethnisch-nationale Identität der Bewohner Oberschlesiens und des Teschener Schlesiens (=Via Silesia. Veröffentlichungen zur deutsch-polnischen Verständigung. Bd. 2). Münster 2008; Erzbischof Alfons Nossol: Schlesien, Śląsk, Slezsko! In: Hałub, Marek/ Weber, Matthias (Hrsg.): Mein Schlesien - meine Schlesier. Zugänge und Sichtweisen (= Schlesische Grenzgänger, Bd. 6). Leipzig 2014. S. 123-129; Szlachta-Misztal, Justyna: Czyistnieje kulturagórnośląska? Rozważania na temat kultury regionu. In: Radomski, Andrzej/ Bomba, Radosław (Hrsg.): Granice w kulturze. Lublin 2010, S. 307-318, hier S. 308.

${ }^{29}$ Siehe z.B.: Nossol, Erzbischof Alfons: Schlesien, Śląsk, Slezsko! In: Hałub, Marek/ Weber, Matthias (Hrsg.): Mein Schlesien - meine Schlesier. Zugänge und Sichtweisen (= Schlesische Grenzgänger, Bd. 6). Leipzig2014. S. 123-129, hier S. 127.

${ }^{30}$ Zyzik, Krzysztof/ Ogiolda, Krzysztof (Hrsg.): Erzbischof Alfons Nossol. Glück in der Liebe. Rückblick auf mein Leben. Sankt Ottilien 2014, S. 157 f., siehe dazu auch: Nossol, 2014, S. 123-129, hier S. 128. 
Schlesiens wahrgenommen. ${ }^{31}$ Ferner tragen sie dazu bei, dass die untersuchte Region als einzigartiges Gebiet empfunden wird. ${ }^{32}$

${ }^{31}$ Gerade bei der Multikulturalität ist der nach der Wende vollzogene Paradigmenwechsel besonders gut sichtbar: Vor 1989 sprach man nicht von der Multikulturalität Schlesiens. Maria Szmeja vermerkt: „Die Lektüre der historischen Publikationen aus dieser Zeit [50er, 60er und 70 er Jahre, MC], die im Westinstitut (Instytut Zachodni) in Posen, im Schlesischen Institut (Instytut Śląski) in Oppeln oder im Schlesischen Wissenschaftlichen Insitut (Śląski Instytut Naukowy) in Kattowitz herausgegeben wurden, präsentiert ein Bild Schlesiens als ein Gebiet der deutsch-polnischen Kämpfe, nicht als ein multikulturelles Land.” (Szmeja, Maria: Socjologiczne przesłanki powstania „,narodu śląskiego". In: Kossakowska-Jarosz, Krystyna (Hrsg.): Śląskość. Siła tradycji i współczesne problemy. Opole 2005, S. 111-123, hier S. 112. [Hervorh. Und Übers. MC]) Nach der Wende wurde die Multikulturalität Schlesiens "entdeckt" und ist zu einem repräsentativen Kulturelement Schlesiens geworden. Dies ist u.a. in den Titeln der wissenschaftlichen Beiträge sichtbar; exemplarisch dazu: Sołdra-Gwiżdż, Teresa: Wielokulturowość Śląska Opolskiego i jegomłodegopokolenia w perspektywiesocjologicznej. In: Lesiuk, Wiesław (Hrsg.): Region jako przyszła struktura europejska. Opole 1992, S. 45-52.

${ }^{32}$ Inwiefern jedoch von einer "Einzigartigkeit" Schlesiens gesprochen werden kann, macht die Autorin in ihrer Doktorarbeit zum Gegenstand einer wissenschaftlichen Reflexion. Erstens vermerkt sie, dass ebenfalls andere Regionen als einzigartig empfunden werden wollen und analysiert diesbezüglich, welche Mechanismen entwickelt werden, um die eigene Einzigartigkeit gegenüber den anderen zu behaupten. Zweitens zeigt die Autorin, dass viele der Elemente, auf die man sich bei der Einzigartigkeit Schlesiens beruft, sich bei einer genauen Analyse als wiederholbare, d.h. auch für weitere Regionen zutreffende oder gar als universelle Eigenschaften enthüllen. Siehe: Czok, Monika 2017, besonders S. 98-145.

Ferner ist aufdie These des schlesischen Journalisten und Kulturwissenschaftlers Michał Smolorz aufmerksam zu machen, dass die meisten jener Elemente, die man als schlesische Eigenart bezeichnet, nicht so „traditionell schlesisch" seien, wie sie empfunden werden. Seines Erachtens wurde das heutige Ansehen Schlesiens medial kreiert und 
Im gleichen Sinne stellt die nächste Metapher die beschriebene Region dar. So wird Schlesien als ein reich gedeckter Tisch mit drei Standbeinen dargestellt, dessen Fundamente (Beine) die polnische, die deutsche und die mährische Kultur sind. Der Stuhl ist nur dann stabil, wenn alle drei Beine vorhanden sind. Wenn ein Bestandteil fehlen würde, könnte er nicht mehr die Rolle erfüllen, für die er geschaffen wurde. ${ }^{33}$ Die Vielfalt der kulturellen Einflüsse fungiert somit als eine unabdingbare Eigenschaft Schlesiens. Zweitens, ein „reich gedeckter Tisch“ erweckt sehr positive Konnotationen: als ein Ort, bei dem man verweilen möchte, als etwas, von dem man kosten möchte. Somit wird die Vielfalt Schlesiens wieder als eine sehr positive Eigenschaft dargestellt.

Im Kontext der Multikulturalität Oberschlesiens und seiner Bewohner wird häufig die Zusammensetzung unterschiedlicher Elemente mittels unterschiedlicher Metapher präsentiert. Ohne auf diese näher einzugehen, zählt die Oppelner Kulturwissenschaftlerin Teresa Smolińska folgende auf: (Ober) Schlesien sei „ein ethnographischer Kessel”, ein „kulturelles Amalgam”, ein „kulturell-ethnischer Schmelztiegel”, ein „bunt geflochtener Behang". ${ }^{34}$ Weitere Bilder stellt Gerard Bartodziej dar: Schlesien wird als ein kultureller Schmelztiegel sowie als ein Mosaik der Bevölkerungsgruppen und Kulturen aufgefasst. ${ }^{35}$ Marcin Wiatr präsentiert Oberschlesien im Hinblick auf die dort vorhandenen verschiedenen und oft verflochtenen

von den Bewohnern zu eigen gemacht. (Smolorz, Michał: Śląsk wymyślony. Katowice 2013.)

${ }^{33}$ Dobrzańska, Andżelika: Mit Edyta Górniak zum Gipfel. In: Wochenblatt vom 3.-9. Februar 2012, S. 1, 12, hier S. 12.

${ }^{34}$ Smolińska, Teresa: Miejsce folkloru w procesie zmian tożsamości narodowej (na przykładzie Śląska Opolskiego). In: Waldemar Gaida u.a.: Ślack w edukacji regionalnej. Strzelce Opolskie 2005. S. 107121, hier S.111. [Übers. MC]

${ }^{35}$ Bartodziej, Gerhard: Niemcy w Polsce - Śląsk ongiś i dzisiaj. In: Mniejszość niemiecka $w$ Polsce. Historia i teraźniejszość(= Polska Niemcy, Heft 1). Warszawa 1995, S. 7-40, hier S. 12. 
Identitätsangebote als „einen Flickenteppich „schwebender” [...] Identitäten oder Doppelidentitäten". ${ }^{36}$ All diesen Metaphern ist gemeinsam, dass mittels Verwendung plakativer Bilder die Vielfalt der Region erfasst und zum Ausdruck gebracht wird.

Die oben aufgeführten Beispiele präsentieren ein durchaus positives - an manchen Stellen beinahe ein idyllisches - Bild Schlesiens. Dabei ist zu vermerken, dass die große Anzahl der Einflüsse, die Schlesien prägen, jedoch nicht immer vorteilhaft bewertet wird. Manchmal wird sie als problematisch empfunden und nicht als Vielfalt, sondern als Verwicklung gedeutet. In diesem Sinne stellt der Soziologe Lech M. Nijakowski die schlesischen Schicksale sowie die Veränderungen in der Auffassung des Schlesientums dar, indem er das Bild einer ,verwickelten Kletterpflanze“ vor Augen ruft. Ihm zufolge ist „das Schicksal der Schlesier [...] einer verwickelten Kletterpflanze ähnlich, die sich aus verschiedenen Trieben zusammensetzt, die oft aus unterschiedlichen Samen gewachsen sind. Heute gehen die Triebe immer mehr auseinander, einige von ihnen verkümmern und verdorren." 37

\section{Das metaphorische Bild von Schlesiern}

Neben den Metaphern, die sich auf Schlesien als Region beziehen, befinden sich ferner jene, die zwecks der Charakteristik der Schlesier als einer ethnischen Gruppe ${ }^{38}$ angeführt werden. Nicht selten werden jene metaphorischen Bilder von den Autoren selbst kreiert.

${ }^{36}$ Wiatr, Marcin: Deutsch sein in Polen. In: Jahrbuch Polen 2016. Minderheiten. Hg. vom Deutschen Polen-Institut Darmstadt, Wiesbaden 2016, S. 61-71, hier S. 67.

${ }^{37}$ Nijakowski, Lech M.: O procesach narodotwórczych na Śląsku. In: Ders. (Hrsg.): Nadciagaja Ślazacy. Czy istnieje narodowość śląka? Warszawa 2004, S. 132-156, hier S. 155. [Übers. MC]

${ }^{38}$ Siehe dazu die Ausführungen in der Dissertation der Autorin: Czok, Monika 2017, S. 87-138. 
So entwickelt der Wirtschaftswissenschaftler Romuald Jończy eine Metapher der Schlesier, in der er sich der Figur der Mutter bedient:

Ich denke, dass man die Schlesier aus Oberschlesien [...] mit Kindern, die zwei Mütter hatten, vergleichen kann. Die erste, die leibliche Mutter, hat in ihrer Jugend Ärger (d.h. Krieg) angestellt und infolge dessen musste sie das Land verlassen. An diese Mutter können sich nur die ältesten „Kinder“ erinnern. Die Zweite ist die Stiefmutter, die das Haus übernahm, dort die Kinder fand und sie großzog und da sie nur ihre Stiefmutter war, war sie für sie nicht so eine Mutter, wie ihre leibliche Mutter es gewesen wäre oder wie sie sich ihre leibliche Mutter vorgestellt hätten. Sie denken auch deswegen so, weil die Stiefmutter in den ersten Jahren (und dabei meine ich die Jahre 1945-48 [...]) besonders viel Strenge hat walten lassen. Und grundlose Schläge vergisst ein Kind nicht so leicht.

Die Beziehung der Schlesier zu beiden Müttern ist eben so, wie sie ist. Sie empfinden eine besonders starke Verbindung zu der leiblichen Mutter, da sie von ihr viele Eigenschaften geerbt haben. Außerdem hat ihre leibliche Mutter sie nicht vergessen und sich stets im Rahmen ihrer Möglichkeiten für ihre Kinder eingesetzt. Sie haben von ihr verschiedene Geschenke bekommen und seit einiger Zeit dürfen die Kinder ihre Mutter besuchen, sogar dort arbeiten. Und, was noch wichtiger ist, sie verdienen dort viel mehr als bei ihrer Stiefmutter. Doch sie wollen nicht bei ihrer leiblichen Mutter wohnen, weil ihre Häuser dort stehen, wo sie von Geburt an gelebt haben.

Auch die Beziehung zur Stiefmutter ist so, wie sie eben ist. Sie sehen ein, dass sie anders als die Stiefmutter sind, doch sie haben nicht vergessen, dass sie eine strenge Mutter war. Und trotzdem wohnen sie bei ihr. Die Kinder und ihre Stiefmutter lernen sich gegenseitig zu verstehen und erkennen viele Gemeinsamkeiten. Inzwischen sind sie erwachsen und können sich in ihrem Haus viel mehr erlauben. Auch das, was sie nicht mehr erklären müssen: welche der Beiden sie für ihre Mutter halten. ${ }^{39}$

${ }^{39}$ Jończy, Romuald: Die Deutschen in Polen nach 1989 - wer waren sie, wer sind sie, oder anders: Identität der deutschstämmigen Bevölkerung und ihre Rolle in der sozialen und wirtschaftlichen Entwicklung der 
In dieser ausführlichen Beschreibung versucht der Autor die Doppelnatur der Schlesier dem Leser näher zu bringen. Inwieweit das dem Autor gelungen ist, mögen die Leser selbst bewerten. $\mathrm{Zu}$ vermerken ist jedoch die Tatsache, dass der Autor die Gruppe nicht kritisch bewertet oder beschuldigt (er schiebt gewissermaßen die Verantwortung für den Stand der Dinge auf die geschichtlichen Ereignisse; damit scheint er zwar nicht zufrieden zu sein, hat sich wohl jedoch damit abgefunden: „es ist, wie es eben ist."). Die Intention des Autors ist somit nicht, die Schlesier negativ darzustellen, sondern eine Erklärung finden zu wollen, woher die Doppelnatur der Schlesier kommt. Das ist anders als bei den nächsten Metaphern, die den Schlesiern gegenüber wertend sind und in ihrer Aussage negativ anmuten, indem sie die Doppelnatur oder die Multikulturalität der Schlesier nachteilig präsentieren.

So wird der Oberschlesier als ein Mensch dargestellt, der immer „zwischen zwei Stühlen sitzt. “40 Diesem Bild zufolge befindet sich der Oberschlesier immer „dazwischen”: zwischen der deutschen und der polnischen Kultur. Er mag sich zahlreiche Gedanken über seine Identität machen sowie in Polen oder in Deutschland nach seiner Verortung suchen - ständig wird er von dem Gefühl eines Mangels geplagt; als ob ein gewisser Teil seiner Seele ,,auf der anderen Seite der Grenze hängen geblieben wäre." 41

Der Metapher von einem zwischen zwei Staaten zerrissenen Schlesier bedient sich auch der Oppelner Pressewissenschaftler

Oppelner Region. In: In Polen ein Deutscher zu sein. Menschenschicksale in Vorträgen und Erinnerungen. Hrsg. vom Verband der deutsche sozial-kulturellen Gesellschaften in Polen, Opole 2011, S. 106-124, hier S. $110 \mathrm{f}$.

${ }^{40}$ Vgl. Bieniasz, Stanisław: Biografia. Zit. nach: Szczepański, Marek S.: Regionalizm górnośląski: los czy wybór? In: Lech M. Nijakowski (Hrsg.): Nadciagaja Ślazacy. Czy istnieje narodowość śląsa? Warszawa 2004, S. 90-115, hier S. 107.

41 Ebd. 
Joachim Glensk. Doch Glensk verleiht ihr einen besonders kräftigen Ton. In seinem ausdrücklich ironischen Bild wird der Schlesier als jener dargestellt, der auf dem einen Knie vor dem polnischen Staat, auf dem anderen Knie vor dem deutschen Staat kniet. ${ }^{42}$ Das sehr plakative Bild stellt diese ethnische Gruppe in durchaus negativem Lichte dar: Der kniende Schlesier wird hier zu einem Diener, wenn nicht sogar zu einem Bettler herabgewürdigt, der dazu noch für den jeweiligen Staat nur auf einem und nicht auf beiden Knien kniet - somit einen nicht loyalen Staatsbürger darstellt.

\section{Fazit}

Um Schlesien als Region und seine Spezifik zu charakterisieren, wird oftmals zu Metaphern gegriffen. Die geographische Lage Schlesiens und seine historische wie gegenwärtige Bindung an die beide Staaten Deutschland und Polen hat zur Folge, dass die Region als eine Brücke zwischen diesen Ländern und Völkern aufgefasst wird. Die Brücke bleibt die meist verbreitete Schlesien beschreibende Metapher.

Eine Reihe weiterer, unterschiedlicher Metaphern, die bei der Charakteristik Schlesiens verwendet werden, zeigen die Vielfalt der Region. Aus der Analyse geht hervor, dass die Versinnbildlichungen größtenteils ein durchaus positives Bild Schlesiens präsentieren. Es ist jedoch zu vermerken, dass die große Anzahl an Einflüssen, die Schlesien prägen, nicht immer vorteilhaft bewertet wird. Wie oben gezeigt wurde, kann ein und dieselbe Eigenschaft positiv oder negativ gedeutet werden (z.B. Multikulturalität, die bereichert oder die Probleme bereitet).

${ }^{42}$ Glensk, Joachim: Organizowanie się mniejszości niemieckiej w latach 1989-1993 w świetle prasy regionalnej na Górnym Śląsku. In: Lis, Michał (Hrsg.): Śląsk Opolski: nadzieje i zagrożenia demokratycznych przemian. Opole 1994, S. 76-88, hier S. 77. 
Ebenso kann die große Zahl der Metaphern als problematisch interpretiert werden. Sie enthüllt nämlich, wie schwierig es ist, die kulturelle Eigenart dieses Raumes zu definieren. Um diese in Worte zu fassen, greifen die Autoren zu vorhandenen Metaphern oder versuchen, eigene - mehr oder weniger zutreffende - Metaphern zu kreieren. Es scheint, dass all jene schlesischen Eigenschaften, Phänomene und weitere Elemente, die sich schwer in Definitionen und Begriffe fassen lassen, mithilfe der Metapher zum Ausdruck gebracht werden sollen.

Abschließend wird noch auf ein Forschungsdesiderat aufmerksam gemacht. Wie in der obigen Ausführung festgestellt wurde, befinden sich unter den Metaphern auch jene, die nicht nur allein auf Schlesien, sondern auch auf andere europäische Regionen zutreffen. Aus wissenschaftlicher Perspektive wäre es interessant, die Verwendung der Metapher bezüglich Schlesiens mit der metaphorischen Darstellung anderer Regionen mit einer wechselvollen Geschichte, etwa Elsass, Oberlausitz, Südtirol und - last but not least - mit Siebenbürgen zu vergleichen.

\section{Literatur}

Bartodziej, Gerhard: Niemcy w Polsce - Śląskongiś i dzisiaj. In: Mniejszośćniemiecka w Polsce. Historia iteraźniejszość (= Polska-Niemcy, H. 1). Warszawa 1995, S. 7-40.

Bieniasz, Stanisław: Schlesien - die dritte Option. In: Deutsche und Polen. 100 Schlüsselbegriffe. Hrsg. v. Ewa Kobylińska, Andreas Lawaty, Stephan Rüdiger. München 1992, S. 540-547.

Conrads, Norbert: Silesiographia oder Landesbeschreibung. In:

Ders.: Deutsche Geschichte im Osten Europas. Schlesien. Berlin 1994, S. 13-36.

Dobrzańska, Andżelika: Mit Edyta Górniak zum Gipfel. In: Wochenblatt. 3.-9. Februar 2012, S. 1, 12.

Glensk, Joachim: Organizowanie się mniejszości niemieckiej w latach 1989-1993 w świetle prasy regionalnej na Górnym 
Śląsku. In: Lis, Michał (Hrsg.): Ślask Opolski: nadzieje i zagrożenia demokratycznych przemian. Opole 1994, S. 76-88.

Jończy, Romuald: Die Deutschen in Polen nach 1989 - wer waren sie, wer sind sie, oder anders: Identität der deutschstämmigen Bevölkerung und ihre Rolle in der sozialen und wirtschaftlichen Entwicklung der Oppelner Region. In: In Polen ein Deutscher zu sein. Menschenschicksale in Vorträgen und Erinnerungen. Hg. vom Verband der deutschen sozial-kulturellen Gesellschaften in Polen, Opole 2011, S. 106-124.

Kroszel, Janusz: Einführung. In: Oberschlesien als Brücke zwischen Polen und Deutschen. Symposium vom 8.-9. Mai 1990 in Opole. Mülheim/Ruhr 1990, S. 3-9.

Lesiuk, Wiesław: Czy Śląsk Opolski może być przykładem regionu na drodze do Europy? In: Ders. (Hrsg.): Region jako przyszła struktura europejska. Materiaty polsko-niemieckiego sympozjum odbytego w Opolu $w$ dniach 13-15 maja 1992 r. Unter der Leitung von Dieter Bach und Krystian Heffner, Opole 1992, S. 112-115.

Nijakowski, Lech M.: O procesach narodotwórczych na Śląsku. In: Ders. (Hrsg.): Nadciagają Ślazacy. Czy istnieje narodowość śląka? Warszawa 2004, S. 132-156.

Nossol, Alfons Erzbischof: Schlesien, Śląsk, Slezsko! In: Hałub, Marek/ Weber, Matthias (Hrsg.): Mein Schlesien meine Schlesier. Zugänge und Sichtweisen (=Schlesische Grenzgänger, Bd. 6), Leipzig 2014, S. 123-129.

Schlesien - eine Brücke in Europa. Aspekte einer Kulturlandschaft in der Mitte Europas. Hg. vom Landesmuseum Schlesien in Görlitz, Görlitz 1993.

Simonides, Dorota: Mit Ślązaka, zit. nach: Ploch, Gregor/ Myszor, Jerzy/ Kucinski, Christine (Hgg.): Die ethnischnationale Identität der Bewohner Oberschlesiens und des Teschener Schlesiens (= Via Silesia. Veröffentlichungen zur deutsch-polnischen Verständigung. Bd. 2). Münster 2008 . 
Smolińska, Teresa: Miejscefolkloru w procesiezmiantożsamo ścinarodowej (na przykładzie Śląska Opolskiego). In: Gaida,Waldemar u.a.: Ślask w edukacji regionalnej. Strzelce Opolskie 2005, S. 107-121.

Sołdra-Gwiżdż, Teresa: Wielokulturowość Śląska Opolskiego i jego młodego pokolenia w perspektywie socjologicznej. In: Wiesław Lesiuk (Hrsg.): Region jako przyszła struktura europejska. Materiały polsko-niemieckiego sympozjum odbytego w Opolu w dniach 13-15 maja 1992 r.,Unter der Leitung von Dieter Bach und Krystian Heffner, Opole 1992, S. 45-52.

Szczepański, Marek S.: Regionalizm górnośląski: los czy wybór? In: Nijakowski, Lech M. (Hrsg.): Nadciagaja Ślązacy. Czy istnieje narodowość śląska? Warszawa 2004, S. 90-115.

Szewczyk, Grażyna Barbara: Die Identität des Oberschlesiers im Umfeld von Ideologie und Mythos. In: Lasatowicz, Maria Katarzyna/ Joachimsthaler, Jürgen (Hrsg.): Nationale Identität aus germanistischer Perspektive. Opole 1998, S. 87-99.

Szlachta-Misztal, Justyna: Czy istnieje kultura górnośląska? Rozważania na temat kultury regionu. In: Radomski, Andrzej; Bomba, Radosław (Hrsg.): Granice w kulturze. Lublin 2010. S. 307-318.

Szmeja, Maria: Socjologiczne przesłanki powstania „narodu śląskiego". In: Kossakowska-Jarosz, Krystyna (Hrsg.): Ślaskość. Sita tradycji i wspótczesne problemy. Opole 2005, S. 111-123.

Szramek, Emil: Ślask jako problem socjologiczny. Katowice 1934.

Vertrag zwischen der Bundesrepublik Deutschland und der Republik Polen über gute Nachbarschaft und freundschaftliche Zusammenarbeit vom 17. Juni 1991, unter: https:// polen.diplo.de/blob/484312/b6dba5931d46bfdbaf72f8dff299a839/vertrag-17-6-1991-data.pdf [30.08.2019]) 
Wiatr, Marcin: Deutsch sein in Polen. In: Jahrbuch Polen 2016. Minderheiten. Hrsg. vom Deutschen Polen-Institut Darmstadt. Wiesbaden 2016, S. 61-71.

Zyzik, Krzysztof/ Ogiolda, Krzysztof (Hrsg.): Erzbischof Alfons Nossol. Glück in der Liebe. Rückblick auf mein Leben. Sankt Ottilien 2014. 